\title{
Effects of Growth Hormone Therapy on carbohydrate metabolism in Spanish Adults with Growth Hormone Deficiency
}

Paola A. Parra Ramírez1, Laura Pérez Fernández¹, Alberto Fernández Martínez¹, Cristina Grande Aragón², Cristina Álvarez-Escolá1

${ }^{1}$ Endocrinology and Nutrition Department. La Paz University Hospital. Madrid, Spain

${ }^{2}$ Clinical Analysis Department. La Paz University Hospital. Madrid, Spain.

BACKGROUND

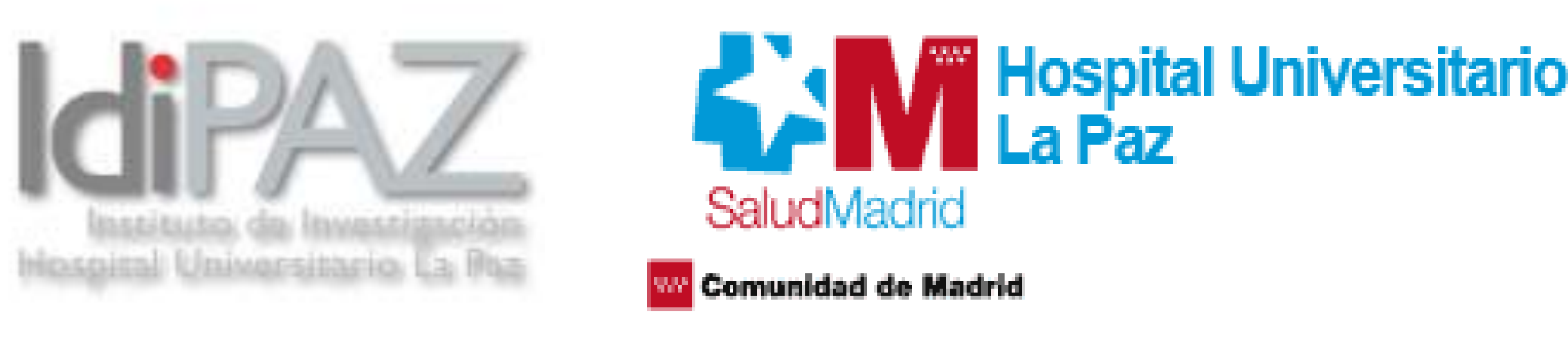

Growth hormone deficiency (GHD) in adults is characterized by a tendency towards fat mass gain and may predispose to type 2 diabetes mellitus. GH replacement (GHR) is associated with impaired insulin sensitivity shortly after starting therapy, reflected by increased fasting glucose and insulin levels. Available evidence suggests that concerns regarding glucose intolerance in patients receiving long-term GHR have not been substantiated. However, several environmental and lifestyle-related factors could influence glucose abnormalities in patients with GHD, and no study has specifically addressed this issue in Spanish patients.

OBJECTIVES

We aimed to describe the evolution of carbohydrate metabolism (fasting glucose -FG- and glycated haemoglobin - $\mathrm{HbA}_{1 \mathrm{c}}{ }^{-}$) and ascertain possible risk factors for developing glucose abnormalities in adult patients receiving GHR.

\section{METHODS}

We analyzed retrospectively $34 \mathrm{GDH}$ adults from our centre who received GHR for at least two years (mean duration of treatment was $7.4 \pm$ 3.5 years ).FG, $\mathrm{HbA}_{1 \mathrm{c}}$ and anthropometric parameters were measured before starting treatment and at the end of the follow-up.

$\mathrm{HbA}_{1 \mathrm{c}}$ was measured by high-performance liquid chromatography. Intra-assay and inter-assay coefficients of variation (CVs) determined using representative blood samples with 5.9 and $10.3 \% \mathrm{HbA}_{1 \mathrm{c}}$ were 0.97 and $0.48 \%$ respectively. Bio-impedance assessment was used to measure total body fat under standard conditions for all measurements.

Associations were tested by Mann-Whitney $U$ test between baseline variables (age, BMI, total body fat, total cholesterol, low-density lipoprotein cholesterol, high-density lipoprotein cholesterol, triglycerides, mean maintenance dose of $\mathrm{GH}$, and glucocorticoid replacement) and these metabolic variables.

\begin{tabular}{|c|c|}
\hline $\begin{array}{c}\text { Baseline } \\
\text { characteristics }\end{array}$ & \\
\hline$N=34$ & \\
\hline Sex: male & 18 \\
\hline $\begin{array}{c}\text { Age } \\
\text { (years) }\end{array}$ & $40.4 \pm 13.5$ \\
\hline $\begin{array}{c}\mathrm{BMI} \\
\left(\mathrm{Kg} / \mathrm{m}^{2}\right)\end{array}$ & $32.9 \pm 13.7$ \\
\hline $\begin{array}{l}\text { Total body fat } \\
\text { (kg/\%) }\end{array}$ & $31.49 \pm 9$ \\
\hline $\begin{array}{c}\text { Total cholesterol } \\
\text { (mg/dl) }\end{array}$ & $215.8 \pm 45.5$ \\
\hline $\begin{array}{l}\text { LDL- cholesterol } \\
\text { (mg/dl) }\end{array}$ & $134.3 \pm 33.4$ \\
\hline $\begin{array}{l}\text { HDL-cholesterol } \\
\text { (mg/dl) }\end{array}$ & $53.75 \pm 14.5$ \\
\hline $\begin{array}{l}\text { Triglycerides } \\
\text { (mg/dl) }\end{array}$ & $112.8 \pm 58.0$ \\
\hline $\begin{array}{l}\text { Fasting glucose } \\
\text { (mg/dl) }\end{array}$ & $87.38 \pm 9.4$ \\
\hline $\begin{array}{c}\mathbf{H b A}_{1 \mathrm{c}} \\
\%\end{array}$ & $5.33 \pm 0.37$ \\
\hline $\begin{array}{l}\text { IGFI } \\
\mathrm{ng} / \mathrm{dl}\end{array}$ & $66.23 \pm 39.5$ \\
\hline
\end{tabular}

\section{RESULTS}

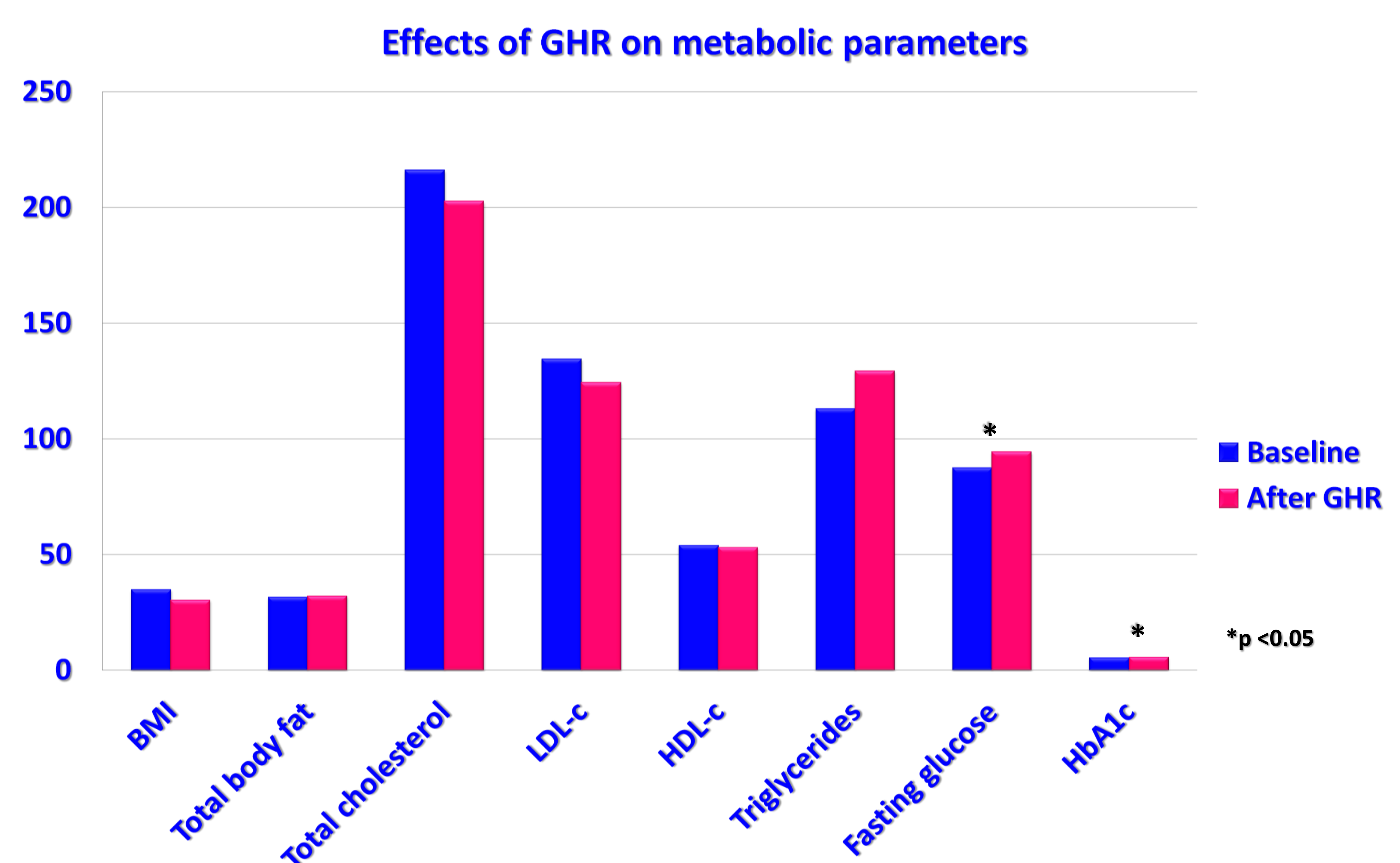

CHANGE IN CARBOHYDRATIC METABOLISM WITH GHR

\% Dysglucosis with GHR

\begin{tabular}{|c|c|c|}
\hline $\begin{array}{c}\text { Fasting } \\
\text { glucose } \\
(\mathrm{mg} / \mathrm{dl})\end{array}$ & $6.7 \pm 11.8$ & $\mathrm{p}=0.001$ \\
\hline $\begin{array}{c}\mathrm{HbA}_{1 \mathrm{c}} \\
\%\end{array}$ & $0.2 \pm 0.4$ & $\mathrm{p}=0.014$ \\
\hline
\end{tabular}

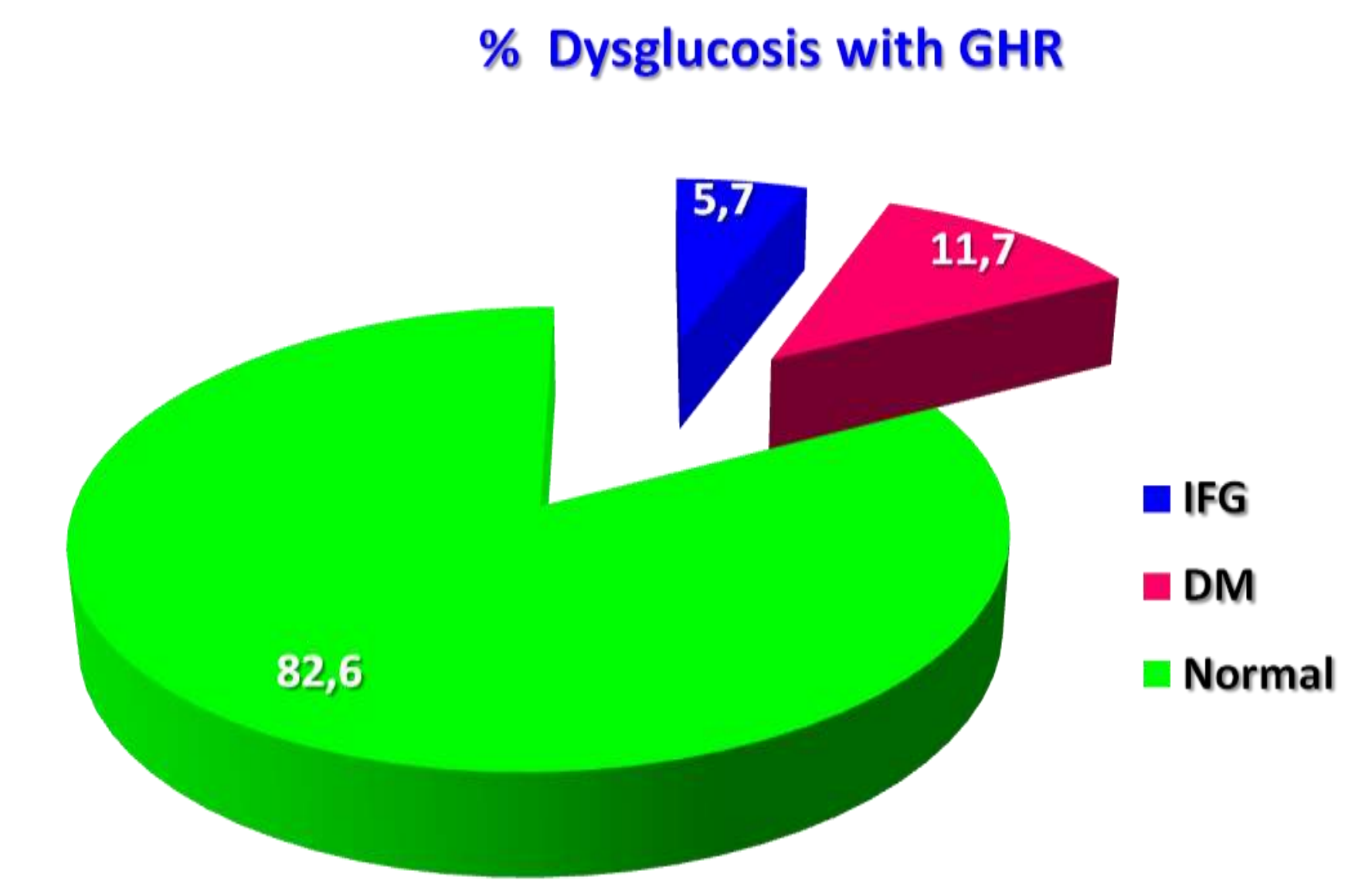

Glucocorticoid replacement and mean maintenance dose of GH $(0.34 \pm 0.2 \mathrm{mg} /$ day $)$ weren't significantly related to the metabolic derangements.

\section{CONCLUSION}

Our results indicate that long-term GHR mildly increases $F G$ and $\mathrm{HbA}_{1 \mathrm{c}}$ in a representative Spanish population. No predefined baseline traits were significantly related to the development of dysglucosis in adult patients receiving standard doses of $\mathrm{GH}$, including glucocorticoid replacement. 